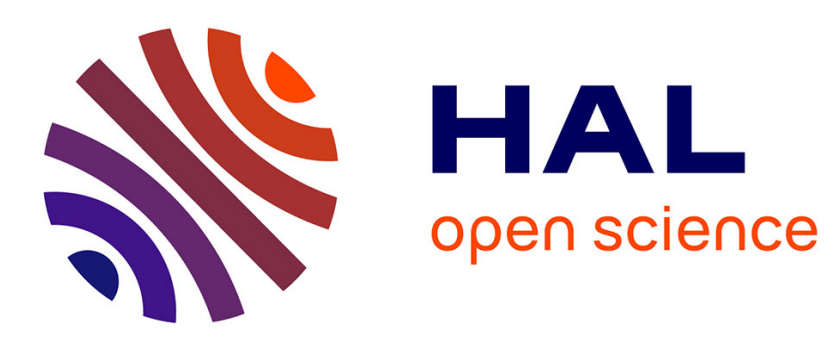

\title{
Les grandes ventes d'automne 1970 dans les forêts soumises au régime forestier
}

\author{
- [.]office National Des Forets, Paris
}

\section{To cite this version:}

- [.]office National Des Forets, Paris. Les grandes ventes d'automne 1970 dans les forêts soumises au régime forestier. Revue forestière française, 1971, 23 (1), pp.5-24. 10.4267/2042/20475 . hal03535030

\section{HAL Id: hal-03535030 \\ https://hal.science/hal-03535030}

Submitted on 19 Jan 2022

HAL is a multi-disciplinary open access archive for the deposit and dissemination of scientific research documents, whether they are published or not. The documents may come from teaching and research institutions in France or abroad, or from public or private research centers.
L'archive ouverte pluridisciplinaire HAL, est destinée au dépôt et à la diffusion de documents scientifiques de niveau recherche, publiés ou non, émanant des établissements d'enseignement et de recherche français ou étrangers, des laboratoires publics ou privés. 


\title{
LES GRANDES VENTES D'AUTOMNE 1970 DANS LES FORÊTS SOUMISES AU RÉGIME FORESTIER
}

Class. Oxford $756: 922$ "1970" (44)

\author{
OFFICE NATIONAL DES FORÊTS
}

Après l'euphorie qui avait caractérisé l'année 1969, on pouvait craindre que les résultats de 1970 ne soient pas aussi satisfaisants. II n'en a rien été et, en ce qui concerne les ventes de bois, 1970 peut être considérée comme une bonne année, venant consolider le rattrapage eflectué en 1969 :

- le marché a absorbé des quantités de bois équivalentes à celles de l'automne dernier (voir annexes I/ et IV, pp. 17 et 24);

- les prix sont en augmentation pour la plupart des produits (voir annexes l et II, pp. 16 et 17), mais sans que ces hausses, dans l'ensemble, s'écartent de la ligne de celles intervenues sur les marchés étrangers ou internationaux.

Deux traits se dégagent particulièrement de ces ventes :

- les résineux ont tenu la vedette : partout, leur demande s'est montrée très pressante, comme en témoigne la hausse des cours nettement plus accentuée que sur les feuillus.

- les acheteurs ont fait preuve d'une séle ction plus sévère dans leurs achats, de telle sorte que la proportion des invendus s'e $n$ est trouvée légèrement accrue, tout en demeurant très sensiblement intérieure à celle des grandes ventes de 1966 à 1968 (voir annexe IV, p. 24).

Le volume global de bois vendu à l'automne 1970 est très voisin de celui atteint lors des dernières grandes ventes : $6270000 \mathrm{~m}^{3}$ contre $6360000 \mathrm{~m}^{3}$ à l'automne précédent. Ce chiffre recouvre, en fait, un volume un peu supérieur en feuillus $(+4 \%)$, un peu moindre en résineux $(-3 \%)$ et en taillis et houppiers tant feuillus que résineux $(-5 \%)$; mais la diminution du volume de rèsineux commercialisés n'est qu'apparente puisqu'il en a été vendu davantage au printemps de cette année qu'au printemps 1969. 
Le volume offert étant un peu supérieur à celui de l'automne 1969, il en découle que les invendus ont été plus nombreux, surtout en ce qui concerne les feuillus $(12 \%$ contre $9 \%$ ) et les taillis et houppiers (16\% contre $12 \%$. Ceci traduit, entre autres, le ralentissement du marché des bois de trituration feuillus qui a commencé à se faire sentir à cette époque.

Ces invendus sont nettement moins localisés qu'à l'automne dernier : en effet, certaines régions, comme l'Aquitaine et la Champagne, où l'on avait enregistré des pourcentages élevés d'invendus aux ventes de 1969, ont vu, cet automne, ces pourcentages se réduire de moitié ; par contre, d'autres régions, comme la Franche-Comté, la Lorraine, la Picardie, la Région parisienne, les Pays de la Loire, le Centre, qui avaient pratiquement vendu la totalité de leur offre l'an passé, comptent quelques invendus cette fois-ci:

Le montant des recettes, avec 322,7 millions de francs a atteint, cet automne, un niveau record. Comme les quantités écoulées ont été équivalentes, voire même un peu inférieures, ce phénomène traduit une certaine hausse des prix. En effet, le calcul du prix moyen du mètre cube de bois fait apparaître une hausse de l'ordre de $12 \%$ par rapport à l'automne 1969 et confirme bien le rattrapage intervenu à cette époque.

\section{TOUTES ESSENCES}

Evolution du prix moyen aux Grandes Ventes

Graphique $n^{\circ} 1$ (Houppiers et taillis exclus - Taxe forfaitaire comprise)

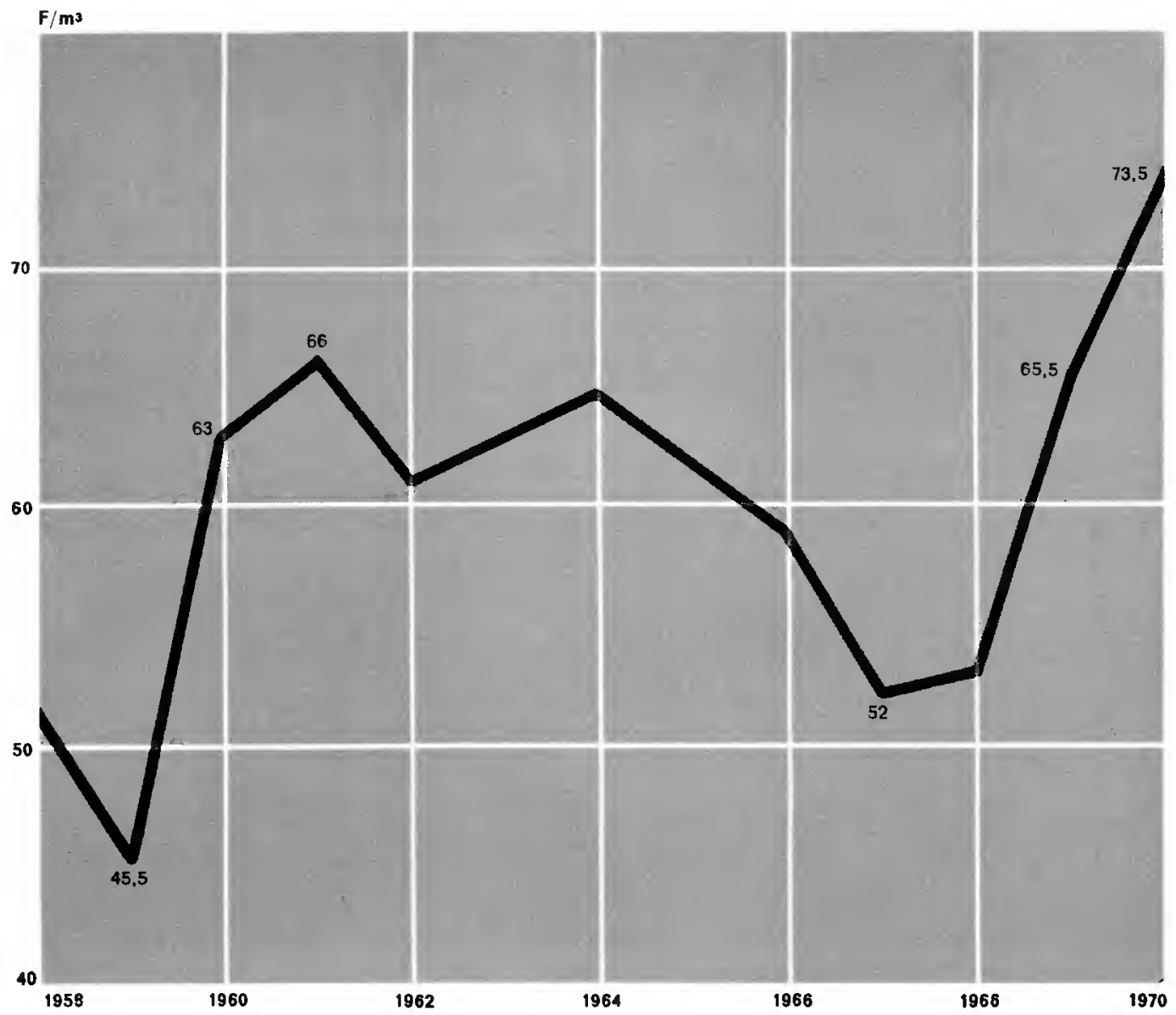


Ces résultats d'ensemble doivent être nuancés : en effet, la hausse s'est montrée, cette année, beaucoup plus sensible sur les résineux $(+18 \%)$ que sur les feuillus $(+6 \%)$. C'est ce qui explique notamment que les recettes des collectivités publiques (152,6 millions de francs) ont davantage progressé que celles des forêts domaniales (170,1 millions de francs), les forêts des collectivités publiques comportant une plus grande proportion de résineux.

Les prix des feuillus n'en sont pas moins demeurés très fermes, comme le montre l'analyse détaillée par essence qui suit.

\section{LE CHENE}

Dans l'ensemble, l'activité du marché du chêne s'est maintenue au même niveau que celui atteint l'an dernier, mais sans marquer de réel progrès.

Malgré une offre un peu supérieure, les volumes vendus ont été quasi identiques à ceux de l'automne 1969 (diagramme $n^{\circ} 1$ ). Le pourcentage des invendus a donc été un peu plus important, particulièrement en ce qui concerne les arbres de petite taille (25 cm et moins de diamètre) et de grosse taille $(50 \mathrm{~cm}$ et plus de diamètre). Toutefois, en ce qui concerne les petits bois, si l'on tient compte du volume accru mis en vente, on constate que le marché en a absorbé davantage en valeur absolue, notamment en Picardie, dans le Centre et en Lorraine.

Diagramme no 1

\section{CHÊNE}

Volume mis en vente et volume vendu (en $1000 \mathrm{~m}^{3}$ )
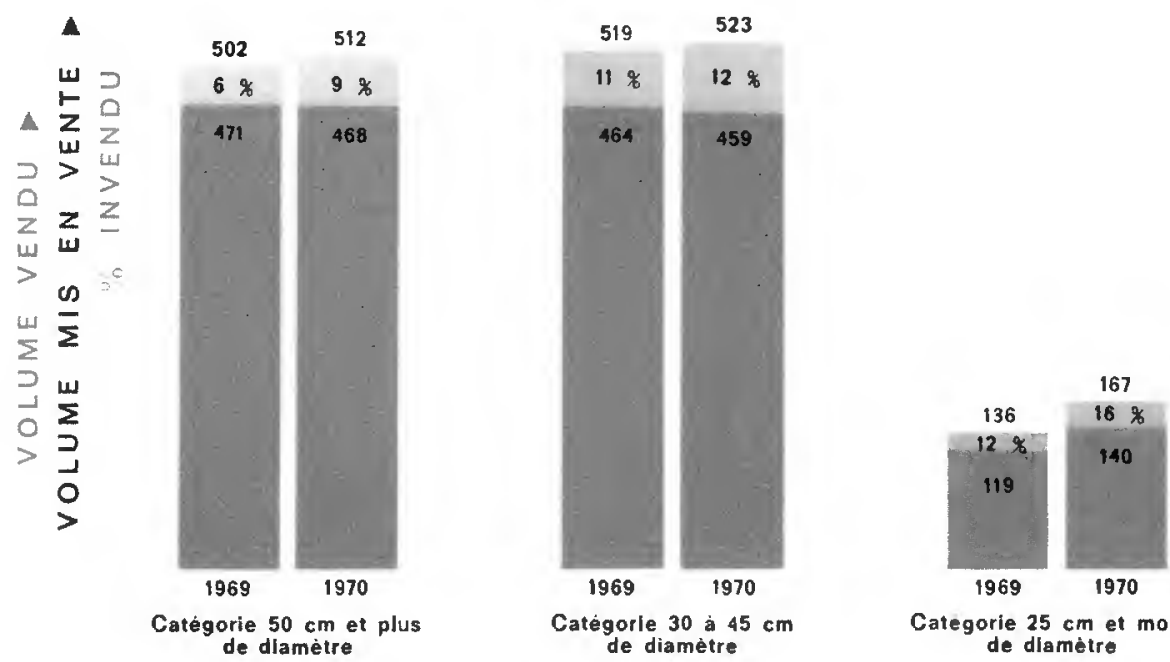

Categorie $25 \mathrm{~cm}$ et moins de diamètre

Le chêne accuse une hausse modérée (graphique $n^{\circ} 2$ ), à peu près égale, qu'il s'agisse des bois de petite, de moyenne ou de grosse dimension ( +7 à $8 \%$ ). Cependant, en raison de la très grande diversité des qualités que peut comprendre la catégorie des chênes de $50 \mathrm{~cm}$ et plus de diamètre, il est très difficile d'en déceler l'évolution réelle. 


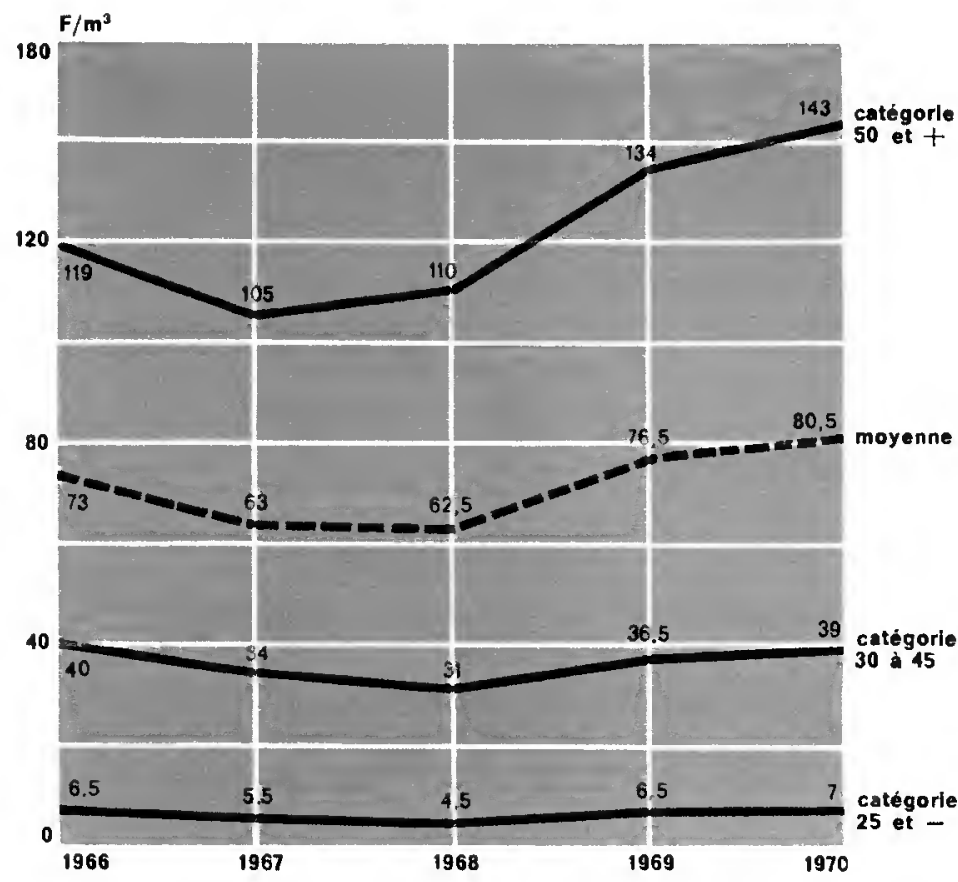

Graphique $n^{\circ} 2$

CHÉNE - Evolution des prix moyens aux Grandes Ventes (taxe forfaitaire comprlse)

Elle semble avoir été assez irrégulière selon les régions : notamment, dans quelques points de vente où la qualité exceptionnelle de certains lots avait entraîné une concurrence très vive (Tours, Saint-Dizier, Nevers, Fontainebleau), il s'est produit des hausses spectaculaires; de même, elle a été assez accentuée ( +12 à $15 \%$ ) dans toutes les ventes de Bourgogne ; sinon, elle n'a guère dépassé 4 ou 5 points dans la plupart des régions productrices. On note même une légère baisse dans l'Allier, due à ce que les chênes dépourvus de tranchage semblent avoir été peu recherchés, sans doute en raison de la diminution de l'activité du Bâtiment dans la région (tableau $n^{\circ} 1$ ).

Tableau $\mathbf{n}^{\circ} \mathbf{1}$

Chêne de $50 \mathrm{~cm}$ et plus de dlamètre

Principaux départements producteurs - Grandes Ventes 1970

\begin{tabular}{|l|c|c|c|c|}
\hline Départements & $\begin{array}{c}\text { Prix moyen } \\
1970(1)\end{array}$ & $\begin{array}{c}\text { Variation } \\
1970-1969\end{array}$ & $\begin{array}{c}\text { Volume vendu } \\
\left(\mathrm{m}^{3}\right)\end{array}$ & $\begin{array}{c}\text { Proportion } \\
\text { d'invendus }\end{array}$ \\
\hline Allier & 241 & $-1,5 \%$ & 22600 & $1 \%$ \\
Loir-et-Cher & 217 & $+2,5 \%$ & 9500 & $0 \%$ \\
Indre & 295 & $0 \%$ & 11400 & $0 \%$ \\
Orne & 181 & $0 \%$ & 12500 & $14 \%$ \\
Nièvre & 155 & $+14 \%$ & 17200 & $6 \%$ \\
Cóte-d'Or & 126 & $+14 \%$ & 41000 & $4 \%$ \\
Haute-Marne & 137 & $+12 \%$ & 29300 & $10 \%$ \\
\hline
\end{tabular}

(1) Taxe forfaitaire comprise.

$\mathrm{Si}$, globalement, la hausse moyenne des arbres de 30 à $45 \mathrm{~cm}$ de diamètre apparaît à peine plus prononcée que celle des gros diamètres $(+8 \%$ contre $+7 \%)$, il n'en est pas de même à l'échelon local où les hausses se montrent souvent très fortes. (Cette divergence s'explique par une répartition régionale différente des volumes vendus des chênes de catégorie intermédiaire). Comme le confirme la proportion d'invendus à peu près inchangée par rapport à l'automne 1969, la demande des arbres 
de qualité moyenne a été relativement plus vive que celle des qualités supérieures. Le développement de l'industrie des palettes et l'ouverture de nouveaux débouchés à l'exportation en donnent sans doute l'explication (tableau $n^{\circ} 2$ ).

Tableau $n^{\circ} 2$

Chêne de 30 à $45 \mathrm{~cm}$ de dlamètre

Principaux départements producteurs - Grandes Ventes 1970

\begin{tabular}{|c|c|c|c|c|}
\hline Départements & $\begin{array}{c}\text { Prix moyen } \\
1970 \text { (1) }\end{array}$ & $\begin{array}{l}\text { Variation } \\
1970-1969\end{array}$ & $\begin{array}{l}\text { Volume vendu } \\
\qquad\left(\mathrm{m}^{3}\right)\end{array}$ & $\begin{array}{l}\text { Proportion } \\
\text { d'invendus }\end{array}$ \\
\hline $\begin{array}{l}\text { Allier } \\
\text { Loir-et-Cher } \\
\text { Indre } \\
\text { Orne } \\
\text { Nièvre } \\
\text { Côte-d'Or } \\
\text { Haute-Marne }\end{array}$ & $\begin{array}{l}47 \\
58 \\
65 \\
53 \\
32 \\
32 \\
33\end{array}$ & $\begin{array}{r}+51 \% \\
+10 \% \\
+11 \% \\
0 \% \\
+15 \% \\
+18 \% \\
-\quad 6 \%\end{array}$ & $\begin{array}{l}38200 \\
14100 \\
14400 \\
20400 \\
19000 \\
22600 \\
20300\end{array}$ & $\begin{array}{ll}0,5 & \% \\
0 & \% \\
0 & \% \\
7 & \% \\
8 & \% \\
9 & \% \\
15 & \%\end{array}$ \\
\hline
\end{tabular}

(1) Taxe forfailalre comprise.

\section{LE HETRE}

Comme pour le chêne, le marché, vu dans son ensemble, est apparu ferme, mais sans plus. Cette tendance générale doit, toutefois, être nuancée.

Le volume offert était en très nette augmentation sur celui de l'automne dernier, et le volume vendu, même s'il n'a pas suivi la même progression, reste supérieur. En particulier, les quantités vendues se sont accrues en Picardie, en Lorraine et en Aquitaine.

La proportion des invendus n'en est pas moins un peu plus élevée qu'en 1969, surtout en ce qui concerne le hêtre de taille intermédiaire (diagramme $n^{\circ} 2$ ).

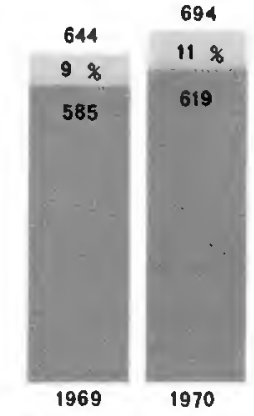

Catégorie $40 \mathrm{~cm}$ et plua de diamètre

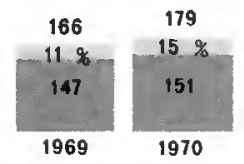

Catégorie 30 oे $35 \mathrm{~cm}$ de diamètre

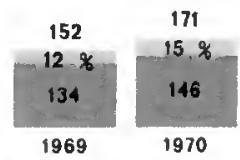

Catégorie $25 \mathrm{~cm}$ et molns de diametre 


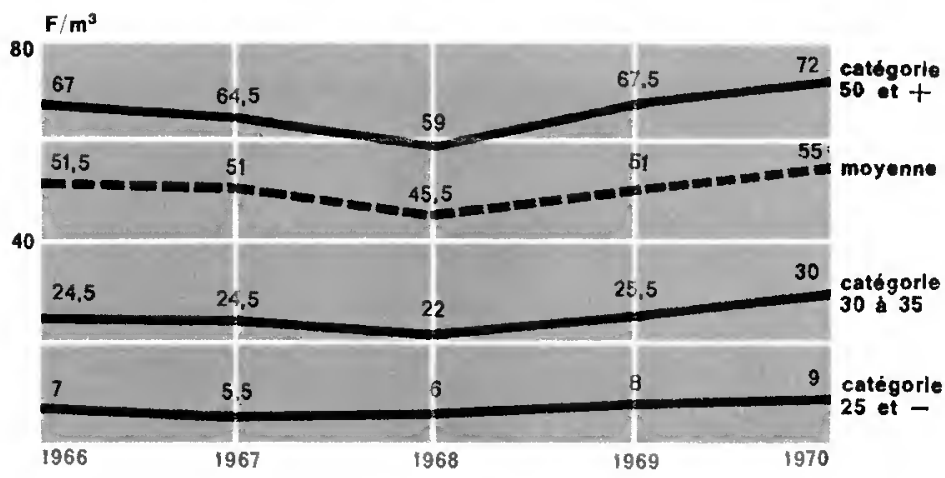

Graphique $n^{\circ} 3$

HÊTRE - Evolution des prix moyens aux Grandes Ventes (taxe fortaire comprise)

Du point de vue des prix (graphique $n^{\circ} 3$ ), la hausse moyenne ressort à $8 \%$, recouvrant une hausse encore plus mesurée pour la catégorie des $40 \mathrm{~cm}$ et plus de diamètre, et au contraire plus accentuée pour les dimensions inférieures.

Cette tendance générale ne reflète pas les évolutions régionales, qui ont été relativement inégales (tableau $n^{\circ} 3$ ). En effet, les prix apparaissent plutôt en baisse $(-2$ à $3 \%)$ en Lorraine, stationnaires en Picardie, en hausse légère $(+4$ à $5 \%)$ en Franche-Comté et en Champagne, en hausse plus accentuée ( +10 à $15 \%$ ) en Normandie, dans les Pyrénées et en Bourgogne.

Tableau $n^{\circ} 3$

Hêtre de $40 \mathrm{~cm}$ et plus de diamètre

Principaux départements producteurs - Grandes Ventes 1970

\begin{tabular}{|l|c|c|c|c|}
\hline Départements & $\begin{array}{c}\text { Prix moyen } \\
1970(1)\end{array}$ & $\begin{array}{c}\text { Variation } \\
1970-1969\end{array}$ & $\begin{array}{c}\text { Volume vendu } \\
\left(\mathrm{m}^{3}\right)\end{array}$ & $\begin{array}{c}\text { Proportion } \\
\text { d'individus }\end{array}$ \\
\hline Seine-Maritime & 84 & $+10 \%$ & 63300 & $8 \%$ \\
Oise & 52 & $-6 \%$ & 46700 & $1 \%$ \\
Aisne & 60 & $+5 \%$ & 37100 & $7 \%$ \\
Haute-Marne & 105 & $+6 \%$ & 31900 & $7 \%$ \\
Meuse & 72 & $-2 \%$ & 40600 & $10 \%$ \\
Basses-Pyrénées & 74 & $+11 \%$ & 31400 & $18 \%$ \\
\hline
\end{tabular}

(1) Taxe forfaitaire comprise.

\section{LES FEUILLUS DIVERS}

L'accroissement des ventes n'a pas suivi le même rythme que celui de l'offre, de telle sorte que la proportion des invendus s'est élevée de façon notable. Le tassement ressenti sur le marché de la trituration donnerait une explication de ces observations.

Tableau $n^{\circ} 4$

Feuillus divers - Volume mis en vente et volume vendu (1000 $\left.\mathrm{m}^{3}\right)$

\begin{tabular}{|c|c|c|c|}
\hline & Volume mis en vente & Volume vendu & $\%$ d'individus \\
\hline 1969 & 298 & 271 & $9 \%$ \\
1970 & 341 & 290 & $15 \%$ \\
\hline
\end{tabular}


Bien que l'hétérogénéïté des produits contenus dans cette rubrique ne permette pas d'en bien suivre les cours, il ne semble pas que leur évolution ait été très différente de celle du chêne ou du hêtre, comme en témoigne la hausse moyenne de $8 \%$.

\section{LE SAPIN ET L'EPICEA}

La diminution du volume de sapin offert et vendu aux ventes d'automne est compensée par le fait qu'il en a été commercialisé davantage au printemps 1970 qu'au printemps 1969.

Le pourcentage des invendus est légèrement plus important ; ces invendus sont presque exclusivement localisés dans les Alpes et les Vosges et sont quasi inexistants dans le Jura et les Pyrénées (diagramme $n^{\circ} 3$ ).

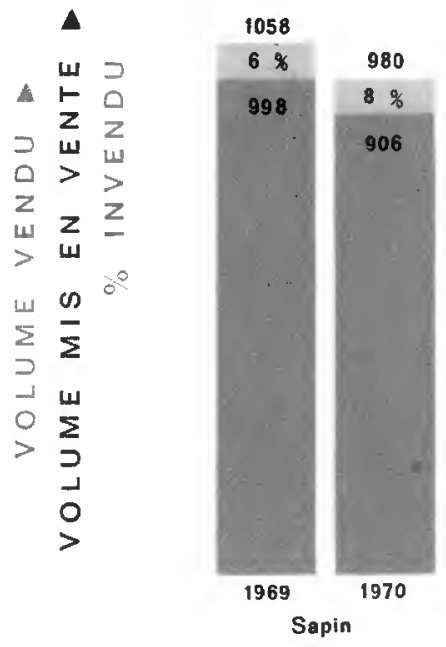

Diagramme $n^{\circ} 3$

SAPIN ET ÉPICÉA

Volume mis en vente

et volume vendu

(en $1000 \mathrm{~m}^{3}$ )

L'augmentation des prix entre 1969 et 1970 est à peine inférieure à celle observée entre 1968 et 1969 (graphique $n^{\circ} 4$ ).

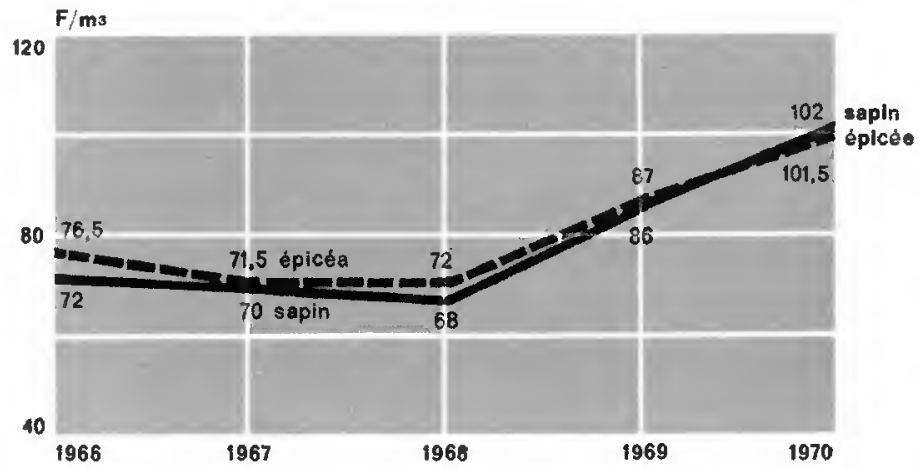

Graphlque n० 4

SAPIN ET ÉPICÉA

Evolution des prix moyens aux Grandes Ventes (taxe forfaitalre comprise) 
Pour la deuxième année consécutive, la hausse se montre plus forte sur le sapin $(+19 \%)$ que sur l'épicéa $(+17 \%)$, de telle sorte que le prix moyen national du sapin, traditionnellement inférieur à celui de l'épicéa, l'a rejoint et même dépassé.

Ceci s'explique pour deux raisons :

- la hausse spectaculaire qui a affecté le sapin de l'Aude et des Pyrénées ;

- la dépréciation relativement accentuée des lots d'exploitation difficile et qui se trouvent être surtout composés d'épicéa.

La hausse des prix a été plus ou moins prononcée selon les régions (tableau $n^{\circ} 5$ ) : ne dépassant guère 10 à $15 \%$ dans les Vosges, en Franche-Comté et en Auvergne, elle a atteint 30 à $40 \%$ dans certains départements alpins et pyrénéens. II est probable que l'amélioration des conditions d'exploitation (développement des réseaux routiers...) a contribué à ce rattrapage des prix vis-à-vis des autres régions moins montagneuses ou mieux desservies.

Tableau $\mathbf{n}^{\circ} 5$

Sapin et Epicéa de plus de $25 \mathrm{~cm}$ de diamètre

Principaux départements producteurs - Grandes Ventes 1970

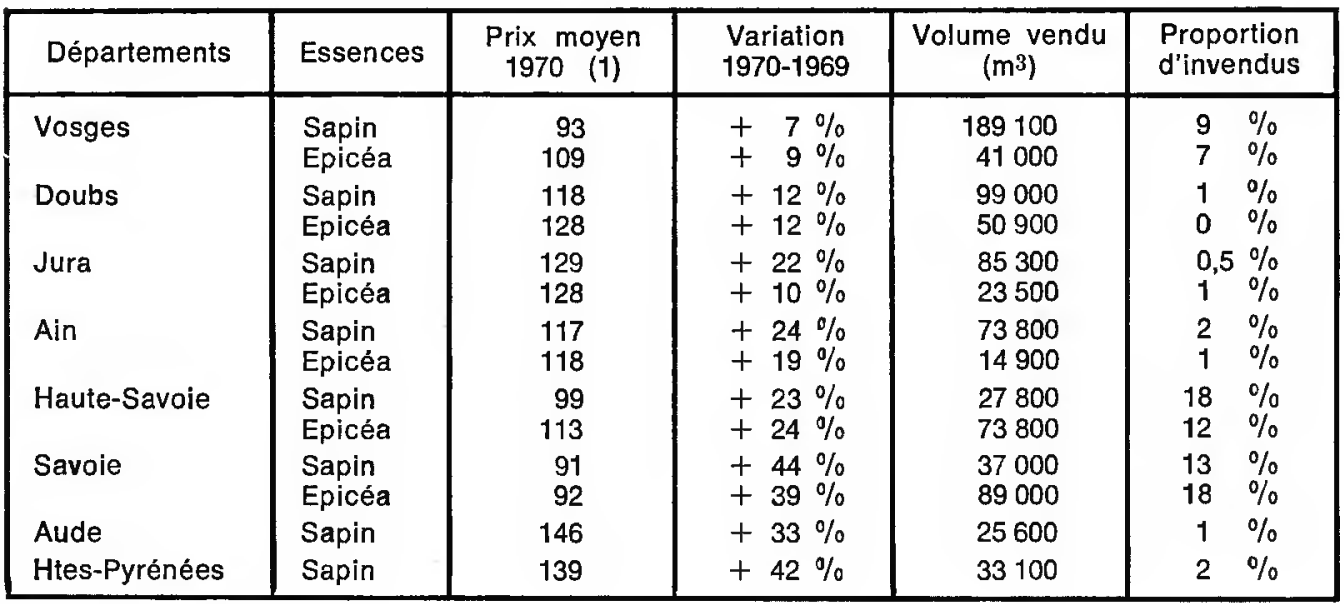

(1) Taxe forfaitaire comprise.

\section{LE PIN SYLVESTRE}

A l'automne, les quantités offertes et vendues de cette essence ont été un peu inférieures à celles de l'automne précédent (tableau $n^{\circ} 6$ ), mais il faut tenir compte de l'important volume qui a été commercialisé pour la première fois au printemps 1970 à Orléans (environ $30000 \mathrm{~m}^{3}$ ).

Tableau n'6

Pin sylvestre - Volume mis en vente et volume vendu (1000 $\left.\mathrm{m}^{3}\right)$

\begin{tabular}{|c|c|c|c|}
\hline & Volume mis en vente & Volume vendu & \% invendu \\
\hline 1969 & 524 & 480 & $8 \%$ \\
1970 & 504 & 447 & $11 \%$ \\
\hline
\end{tabular}




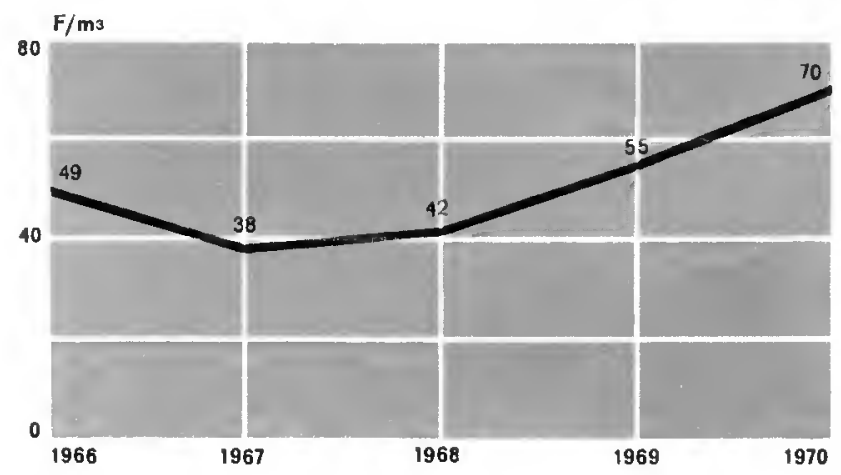

Graphique $n^{\circ} 5$

PIN SYLVESTRE

Evolution des prix moyens aux Grendes Ventes (taxe forfaitaire comprise)

Comme l'an dernier, le pin sylvestre a été très recherché, de telle sorte que son prix a, une nouvelle fois, augmenté de façon assez impressionnante $(+28 \%$ ) (graphique $\left.\mathrm{n}^{\circ} 5\right)$.

L'utilisation de plus en plus courante de cette essence dans la construction et sa raréfaction dans les forêts privées en sont sans doute la cause.

La hausse s'est montrée forte dans la plupart des départements producteurs (tableau $n^{\circ} 7$ ). Et si elle apparaît modérée en Seine-et-Marne, c'est uniquement parce que les qualités offertes cet automne étaient très médiocres.

Tableau $n^{\circ} 7$

Pin sylvestre de $25 \mathrm{~cm}$ et plus de dlamètre

Principaux départements producteurs - Grandes Ventes 1970

\begin{tabular}{|l|c|c|c|c|}
\hline Départements & $\begin{array}{c}\text { Prix moyen } \\
1970(1)\end{array}$ & $\begin{array}{c}\text { Variation } \\
1970-1969\end{array}$ & $\begin{array}{c}\text { Volume vendu } \\
\left(\mathrm{m}^{3}\right)\end{array}$ & $\begin{array}{c}\text { Proportion } \\
\text { d'invendus }\end{array}$ \\
\hline Loiret & 97 & $+44 \%$ & 51900 & 0 \\
Seine-et-Marne & 85 & $+3 \%$ & 10500 & 0 \\
Orne & 114 & $+35 \%$ & 31800 & $0,5 \%$ \\
Seine-Mariłime & 105 & $+60 \%$ & 24900 & $2 \%$ \\
Puy-de-Dôme & 83 & $+30 \%$ & 19300 & 6 \\
Vosges & 51 & $+20 \%$ & 25100 & $15 \%$ \\
\hline
\end{tabular}

(1) Taxe forfaitaire comprise.

\section{LE PIN MARITIME}

A une offre équivalente a correspondu une demande accrue, de telle sorte que, à l'inverse de ce qui s'est passé pour toutes les autres essences -, la proportion des invendus s'est réduite (tableau $n^{\circ} 8$ ).

Tableau $n^{\circ} 8$

PIn maritime - Volume mis en vente ef volume vendu (1000 $\left.\mathrm{m}^{3}\right)$

\begin{tabular}{|c|c|c|c|}
\hline & Volume mis en vente & Volume vendu & $\%$ invendu \\
\hline 1969 & 314 & 263 & $16 \%$ \\
1970 & 315 & 284 & $10 \%$ \\
\hline
\end{tabular}




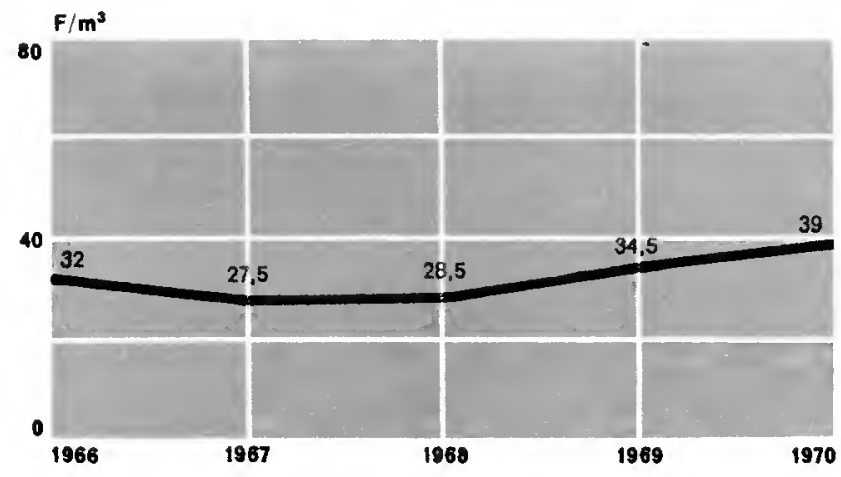

La hausse moyenne de $13 \%$ recouvre une hausse plus marquée (+ 18 à $20 \%$ ), masquée par le fait qu'il a été écoulé cette année des quantités sensiblement plus importantes d'arbres de petits diamètres (graphique $n^{\circ} 6$ ).

La hausse s'est montrée plus forte en Gironde que dans les Landes, contrairement à l'automne précédent (tableau $n^{\circ} 9$ ).

Tableau $n^{\circ} 9$

Pin marltime de $25 \mathrm{~cm}$ et plus de diametre

Principaux départements producteurs - Grandes Ventes 1970

\begin{tabular}{|l|c|c|c|c|}
\hline \multicolumn{1}{|c|}{ Départements } & $\begin{array}{c}\text { Prix moyen } \\
1970(1)\end{array}$ & $\begin{array}{c}\text { Variation } \\
1970-1969\end{array}$ & $\begin{array}{c}\text { Volume vendu } \\
\left(\mathrm{m}^{3}\right)\end{array}$ & $\begin{array}{c}\text { Proportion } \\
\text { d'invendus }\end{array}$ \\
\hline Gironde & 42 & $+23 \%$ & 76200 & $11 \%$ \\
Landes & 48 & $+17 \%$ & 81500 & $11 \%$ \\
\hline
\end{tabular}

(1) Taxe forfaitaire comprise.

\section{LES RESINEUX DIVERS}

Si l'offre a été nettement plus importante qu'à l'automne 1969, la demande s'est aur contraire montrée équivalente, de telle sorte que les volumes vendus sont juste un peu supérieurs et que le pourcentage des invendus s'est sensiblement accru (tableau $n^{\circ}$ 10).

Tableau $n^{\circ} 10$

Résineux divers - Volume mis en vente et volume vendu $\left(1000 \mathrm{~m}^{3}\right)$

\begin{tabular}{|c|c|c|c|}
\hline & Volume mis en vente & Volume vendu & $\%$ invendu \\
\hline 1969 & 279 & 229 & $18 \%$ \\
1970 & 317 & 237 & $25 \%$ \\
\hline
\end{tabular}

Le mouvement des prix ne peut être observé qu'à l'échelon local, étant donné la diversité des essences que renferme cette rubrique (tableau $n^{\circ} 11$ ).

Le mélèze paraît avoir suivi les tendances générales observées pour les résineux ; il n'en est pas tout à fait de même pour le pin noir et le pin à crochets, qui accusent 
des hausses plus modérées ( +8 à $12 \%$, voire même une baisse dans les Alpes de Haute Provence). La forte hausse enregistrée en Lozère est en partie due à une qualité meilleure. A l'inverse, la baisse enregistrée dans la Drôme n'est qu'apparente et s'explique par la qualité très médiocre des lots. En Corse, la hausse spectaculaire du pin laricio n'est que partiellement significative, le prix moyen de l'an dernier ayant été faussé par la commercialisation à très bas prix de lots d'exploitation très difficile.

Résineux divers de $25 \mathrm{~cm}$ et plus de dlamètre Principaux départements producteurs - Grandes Ventes 1970

\begin{tabular}{|l|c|c|c|c|}
\hline \multicolumn{1}{|c|}{ Départements } & $\begin{array}{c}\text { Prix moyen } \\
1970(1)\end{array}$ & $\begin{array}{c}\text { Variation } \\
1970-1969\end{array}$ & $\begin{array}{c}\text { Volume vendu } \\
\left(\mathrm{m}^{3}\right)\end{array}$ & $\begin{array}{c}\text { Proportion } \\
\text { d'invendus }\end{array}$ \\
\hline $\begin{array}{l}\text { Hautes-Alpes (2) } \\
\text { Alpes de Haute }\end{array}$ & 48 & $+13 \%$ & 37200 & $25 \%$ \\
Provence (3) & 36 & $-10 \%$ & 23600 & $26 \%$ \\
Drôme (4) & 18 & $-8 \%$ & 8800 & $27 \%$ \\
Lozère (5) & 53 & $+25 \%$ & 14600 & $24 \%$ \\
Pyrénées-Orientales (6) & 42 & $+8 \%$ & 18800 & $23 \%$ \\
Corse (7) & 107 & $+52 \%$ & 21200 & $29 \%$ \\
\hline
\end{tabular}

(1) Taxe forfaitaire comprise.

(2) Essentiellement mélèze et pin noir.

(4) Uniquement pin noir.

(3) Essentiellement pin noir, accessoirement

(5) Essentiellement pin noir et pin à crochets. pin à crochets et mélèze.

(6) Uniquement pln à crochets.

(7) Uniquement pin laricio.

\section{CONCLUSIONS}

Malgré la très nette amélioration de la situation du marché du bois et la sensible revalorisation des cours intervenues en 1969 et 1970, force est de constater que l'évolution des prix moyens des bois ne compense pas, sur une longue période, l'érosion monétaire, puisqu'à l'automne ces prix dépassaient à peine, en francs courants, ceux pratiqués au cours des grandes ventes de 1961 (voir annexe I).

Ainsi l'équilibre de l'économie sylvicole paraît à terme, compromis. II est donc plus utile que jamais de rechercher tant l'accroissement de la productivité — par un abaissement progressif des coûts et une lente augmentation des rendements en quantité comme en qualité - que la valorisation des services d'intérêt général rendus par la forêt à l'époque de l'environnement.

DIRECTION ÉCONOMIQUE OFFICE NATIONAL DES FORETTS

4. avenue de Saint-Mandé PARIS - $12^{\circ}$ 


\section{ANNEXE I}

\section{ÉVOLUTION DES PRIX MOYENS OBTENUS AUX GRANDES VENTES 1970}

\section{(Taxe forfaitaire comprise)}

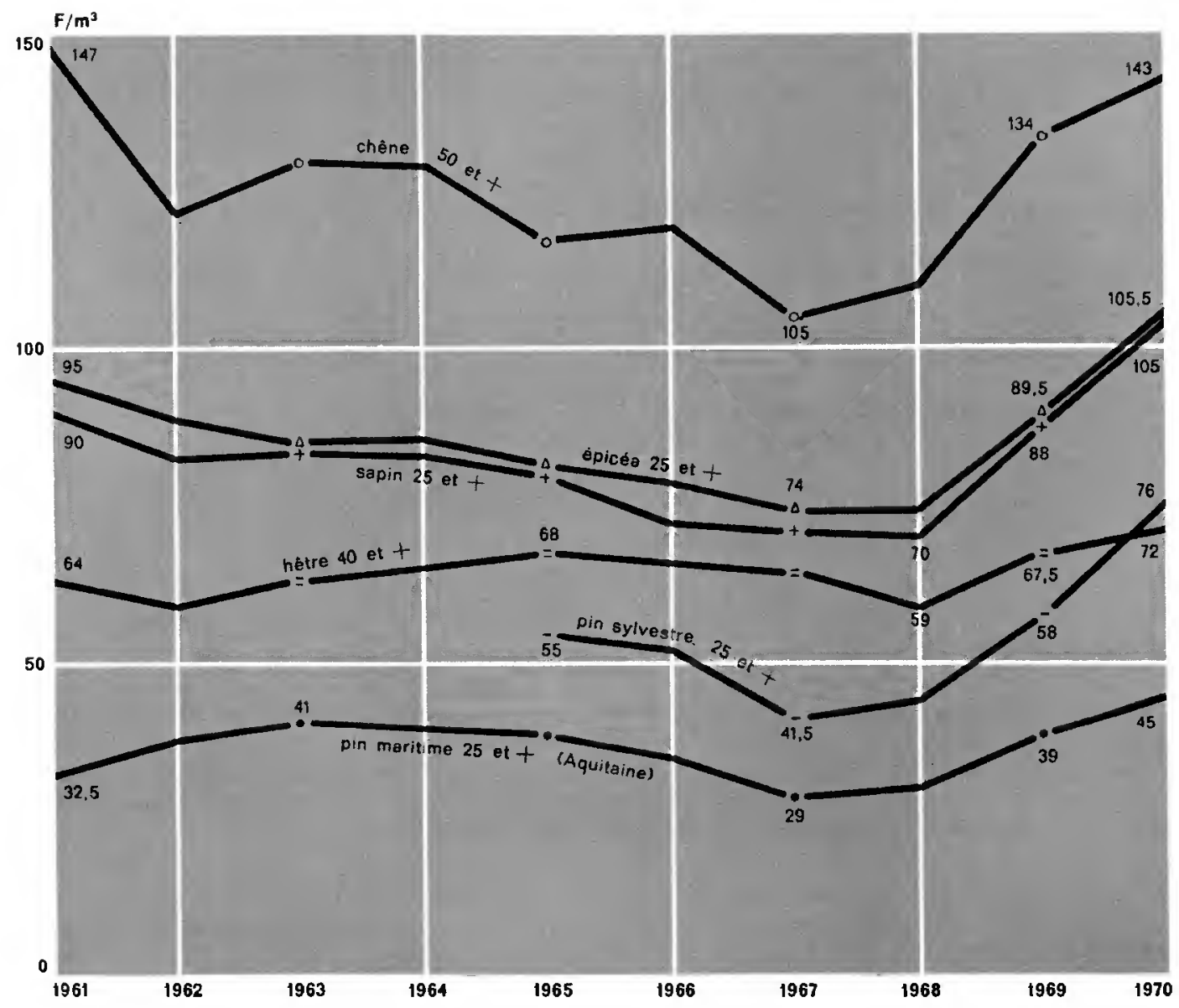

Nota : Le changement de classification des produits présumés des coupes entre 1964 et 1965 e posé un probléme de raccordement des catégories de dimension retenues pour chaque essence, afin de pouvoir suivre les prlx au cours d'une période de temps suffisamment longue. Si le chene de $160 \mathrm{~cm}$ et plus de circonférence correspondait essez blen à celui de $50 \mathrm{~cm}$ et plus de diamétre et si les résineux de qualité sciage et charpente se retrouvent essentlellement dans la categorie de $25 \mathrm{~cm}$ et plus de diamétre, il n'en était pas de méme pour le hétre : le hêtre de $120 \mathrm{~cm}$ et plus de circonférence était légèrement Inférieur à celui de 40 cm et plus de diametre, retenu dans la nouvelle classification de 1965 ; les prix du hétre antérieurs à 1965 ont donc été augmentés de $5 \%$ pour tenir comple du léger décalage des dimensions. 


\section{GRANDES VENTES 1970}

\begin{tabular}{|c|c|c|c|c|c|c|}
\hline Essences & $\begin{array}{c}\text { Volume } \\
\text { mis en } \\
\text { vente } \\
\left(\begin{array}{llll}1 & 000 \mathrm{~m}^{3}\end{array}\right)\end{array}$ & $\begin{array}{c}\text { Volume } \\
\text { vendu } \\
\left(1000 \mathrm{~m}^{3}\right)\end{array}$ & $\begin{array}{c}\text { Volume } \\
\text { invendu } \\
\left(1000 \mathrm{~m}^{3}\right)\end{array}$ & $\begin{array}{c}\text { Prix } \\
\text { moyen } \\
1970(1) \\
\left(\mathrm{F} / \mathrm{m}^{3}\right)\end{array}$ & $\begin{array}{c}\text { Prix } \\
\text { moyen } \\
1969(1) \\
\left(\mathrm{F} / \mathrm{m}^{3}\right)\end{array}$ & $\begin{array}{l}\text { Variation } \\
1970-1969\end{array}$ \\
\hline Chêne 50 et $+\ldots \ldots$ & 512 & 468 & 44 & 143 & 134 & $+\quad 7 \%$ \\
\hline Chêne $30-45 \ldots \ldots \ldots$ & 523 & 458 & 65 & 39 & 36,5 & $8 \%$ \\
\hline Chêne 25 et $-\ldots \ldots$ & 167 & 140 & 27 & 7 & 6,5 & $9 \%$ \\
\hline Total chêne .......... & 1202 & 1066 & 136 & 80,5 & 76,5 & $5 \%$ \\
\hline Hêtre 40 et $+\ldots \ldots$. & 694 & 619 & 75 & 72 & 67,5 & $+7 \%$ \\
\hline Hêtre $30-35 \ldots \ldots$. & 179 & 151 & 28 & 30 & 25,5 & $+17 \%$ \\
\hline Hêtre 25 et $-\ldots \ldots$. & 171 & 146 & 25 & 9 & 8 & $+13 \%$ \\
\hline Total hêtre....... & 1044 & 916 & 128 & 55 & 51 & $+8 \%$ \\
\hline Peuplier $\quad . . . . . . . . .$. & 18 & 17 & 1 & 75 & 63,5 & $+18 \%$ \\
\hline Autres feuillus...$\ldots$. & 341 & 290 & 51 & 19,5 & 18 & $+8 \%$ \\
\hline Total feuillus ........ & 2605 & 2289 & 316 & 62,5 & 59 & $+6 \%$ \\
\hline Sapin 25 et $+\ldots \ldots$. & 944 & 874 & 70 & 105 & 88 & $+19 \%$ \\
\hline Sapin 20 et $-\ldots \ldots$. & 36 & 32 & 4 & 34,5 & 29,5 & $+18 \%$ \\
\hline Total sapin .......... & 980 & 906 & 74 & 102 & 86 & $+19 \%$ \\
\hline Epicéa 25 et $+\ldots \ldots$ & 517 & 463 & 54 & 105,5 & 89,5 & $+17 \%$ \\
\hline Epicéa 20 et $-\ldots$. & 30 & 26 & 4 & 31 & 27 & $+14 \%$ \\
\hline Total épicéa....... & 547 & 489 & 58 & 101,5 & 87 & $+17 \%$ \\
\hline Pin sylvestre 25 et + & 451 & 404 & 47 & 76 & 58 & $+31 \%$ \\
\hline Pin sylvestre 20 et - & 53 & 43 & 10 & 18,5 & 16 & $+16 \%$ \\
\hline Total pin sylvestre .... & 504 & 447 & 57 & 70 & 55 & $+28 \%$ \\
\hline Pin maritime 25 et + & 229 & 204 & 25 & 47,5 & 39,5 & $+20 \%$ \\
\hline Pin maritime 20 et - & 86 & 80 & 6 & 17 & 14,5 & $+19 \%$ \\
\hline Total pin maritime .... & 315 & 284 & 31 & 39 & 34,5 & $+13 \%$ \\
\hline Autres résineux 25 et + & 242 & 183 & 59 & 52,5 & 43 & $+22 \%$ \\
\hline Autres résineux 20 et - & 75 & 54 & 21 & 15 & 16 & - $6 \%$ \\
\hline Total autres résineux .. & 317 & 237 & 80 & 44 & 37 & $+18 \%$ \\
\hline Total résineux $\ldots . .$. & 2662 & 2362 & 300 & 82,5 & 70 & $+18 \%$ \\
\hline $\begin{array}{l}\text { Taillis et houppiers } \\
\text { feuillus } \\
\text {............ }\end{array}$ & 1716 & 1429 & 287 & 2,2 & 2 & $+7 \%$ \\
\hline Houppiers résineux ... & 222 & 191 & 31 & 0,2 & 0,1 & $+100 \%$ \\
\hline Total gėnėral & 7205 & 6271 & 934 & 54,5 & 48 & $+14 \%$ \\
\hline
\end{tabular}

(1) Taxe forfaitaire comprise. 


\begin{tabular}{|c|c|c|c|c|c|c|c|c|c|c|c|c|c|}
\hline \multirow{3}{*}{ Départements } & \multicolumn{4}{|c|}{ Chêne } & \multicolumn{4}{|c|}{ Hêtre } & \multicolumn{2}{|c|}{ Autres feuillus } & \multicolumn{3}{|c|}{ Sapin } \\
\hline & \multicolumn{3}{|c|}{$\begin{array}{l}\text { Prix moyens } \\
\qquad(\mathrm{F} / \mathrm{m} 3)\end{array}$} & \multirow{2}{*}{$\begin{array}{c}\begin{array}{c}\text { Volume } \\
\text { vendu } \\
\text { (m3) }\end{array} \\
\overline{\overline{\widetilde{J}}} \\
\end{array}$} & \multicolumn{3}{|c|}{$\begin{array}{c}\mathrm{Prlx} \text { mayens } \\
(\mathrm{F} / \mathrm{m} 3)\end{array}$} & \multirow{2}{*}{$\begin{array}{c}\begin{array}{c}\text { Volume } \\
\text { vendu } \\
(\mathrm{m} 3)\end{array} \\
\overline{\overline{0}} \\
5\end{array}$} & \multirow[t]{2}{*}{$\begin{array}{l}\text { Prix } \\
\text { moyens } \\
(\mathrm{F} / \mathrm{m} 3)\end{array}$} & \multirow{2}{*}{$\begin{array}{c}\begin{array}{c}\text { Volume } \\
\text { vendu } \\
\text { (m3) }\end{array} \\
\overline{\frac{\pi}{0}} \\
\text { i }\end{array}$} & \multicolumn{2}{|c|}{$\begin{array}{l}\text { Prix moyens } \\
(\mathrm{F} / \mathrm{m} 3)\end{array}$} & \multirow{2}{*}{ 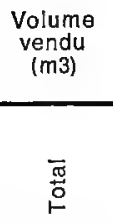 } \\
\hline & $\begin{array}{l}+ \\
\widetilde{\Phi} \\
\stackrel{8}{n}\end{array}$ & 号 & $\begin{array}{l}1 \\
\square \\
\mathbb{\alpha} \\
\mathbb{N}\end{array}$ & & $\begin{array}{l}+ \\
\vec{\Phi} \\
g\end{array}$ & 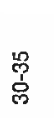 & $\begin{array}{l}1 \\
0 \\
20\end{array}$ & & & & $\begin{array}{l}+ \\
+ \\
\mathbb{\Phi} \\
\stackrel{4}{\Delta}\end{array}$ & $\begin{array}{l}1 \\
\overrightarrow{0} \\
\stackrel{\alpha}{ }\end{array}$ & \\
\hline 1 Ain & 52 & 23 & - & 5400 & 50 & 24 & 5 & 9200 & 18 & 2100 & 110 & 27 & 74300 \\
\hline 2 Aisne & 110 & 30 & 6 & 28500 & 57 & 20 & 10 & 42900 & 22 & 27900 & - & 一 & - \\
\hline 3 Allier & 227 & 44 & 4 & 70600 & 107 & 41 & 8 & 18800 & 13 & 2800 & 144 & 12 & 1300 \\
\hline 4 Basses-Alpes & - & - & - & - & - & - & 一 & 200 & 一 & - & 42 & 22 & 6300 \\
\hline 5 Hautes-Alpes & - & 一 & - & - & 16 & 12 & 5 & 1400 & - & 一 & 77 & 35 & 15500 \\
\hline 6 Alpes-Maritimes & - & - & - & 一 & - & 一 & 一 & - & 一 & - & 87 & 17 & 16800 \\
\hline 7 Ardèche & - & 一 & 一 & 一 & 12 & 6 & 3 & 3500 & - & - & 107 & 53 & 13400 \\
\hline 8 Ardennes & 106 & 28 & 4 & 25500 & 53 & 16 & 6 & 8300 & 25 & 12900 & - & 一 & - \\
\hline 9 Ariège & 95 & 23 & 3 & 1200 & 33 & 21 & 3 & 35400 & - & 400 & 99 & 30 & 14200 \\
\hline 10 Aube & 96 & 34 & 3 & 17300 & 71 & 25 & 5 & 5200 & 25 & 3600 & - & 一 & 一 \\
\hline 11 Aude & 100 & 60 & 10 & 1000 & 121 & 80 & 15 & 8000 & - & 500 & 138 & 45 & 26138 \\
\hline 12 Aveyron & 一 & 一 & 一 & - & 72 & 38 & 7 & 5000 & - & - & - & 一 & 600 \\
\hline 13 B.-du-Rhône & - & 一 & - & - & - & 一 & 一 & - & - & - & - & 一 & - \\
\hline 14 Calvados & 50 & 24 & 14 & 3300 & 80 & 37 & 22 & 9200 & - & - & - & 一 & - \\
\hline 15 Cantal & 44 & 30 & 6 & 900 & 24 & 12 & 2 & 2900 & 10 & 800 & 117 & 15 & 10300 \\
\hline 16 Charente & 207 & 41 & 7 & 3900 & 一 & - & - & 300 & 11 & 1000 & - & 一 & - \\
\hline 17 Char.-Maritime & 一 & 一 & 一 & 700 & - & - & - & - & 一 & - & 一 & - & - \\
\hline 18 Cher & 192 & 31 & 4 & 22200 & 60 & 30 & 2 & 2100 & 4 & 1400 & 一 & - & - \\
\hline 19 Corrèze & - & - & 一 & 100 & 一 & - & 一 & 一 & 一 & 100 & - & 一 & - \\
\hline 20 Corse & 一 & - & 一 & - & 一 & 一 & 一 & 一 & - & - & 86 & 一 & 1200 \\
\hline 21 Côte-d'Or & 118 & 30 & 3 & 67400 & 75 & 22 & 4 & 27600 & 20 & 16700 & - & 一 & - \\
\hline 22 Côtes-du-Nord & - & - & - & 300 & 66 & 31 & 10 & 1400 & - & - & 一 & - & - \\
\hline 23 Creuse & 65 & 29 & 6 & 2300 & 74 & 35 & 10 & 2700 & 一 & 400 & - & 一 & - \\
\hline 24 Dordogne & - & - & 一 & - & - & - & - & - & - & - & - & - & - \\
\hline 25 Doubs & 73 & 27 & 4 & 18800 & 80 & 27 & 10 & 26400 & 26 & 10500 & 111 & 36 & 102000 \\
\hline 26 Drôme & - & 一 & - & 300 & 52 & 41 & 8 & 13000 & 一 & 200 & 77 & 20 & 32300 \\
\hline 27 Eure & 81 & 40 & 10 & 5800 & 80 & 45 & 19 & 18300 & 41 & 1900 & - & 一 & 600 \\
\hline 28 Eure-et-Loir & 240 & 60 & 3 & 12700 & 75 & 42 & 3 & 2100 & - & - & - & 一 & - \\
\hline 29 Finistère & 79 & 37 & 5 & 1300 & 47 & 27 & 9 & 700 & 一 & - & 一 & 一 & - \\
\hline 30 Gard & - & - & 一 & 300 & 14 & 14 & 2 & 5600 & 7 & 2700 & - & 一 & 700 \\
\hline 31 Haute-Garonne & 95 & 40 & 8 & 1100 & 48 & 27 & 4 & 16000 & - & 300 & 84 & 16 & 22400 \\
\hline 32 Gers & 91 & 87 & 6 & 900 & - & - & 一 & - & - & - & - & - & - \\
\hline
\end{tabular}

Remarques

1. Les prix moyens s'entendent taxe forfaitaire exclue.

2. Les prix moyens et volumes vendus ne sont calculés que pour les volumes-tiges inventoriés. 
SOUMISES AU REGIME FORESTIER

sur pied vendus aux Grandes Ventes 1970

\begin{tabular}{|c|c|c|c|c|c|c|c|c|c|c|c|c|}
\hline \multicolumn{3}{|c|}{ Epicéa } & \multicolumn{3}{|c|}{ Pin sylvestre } & \multicolumn{3}{|c|}{ Pin marttime } & \multicolumn{3}{|c|}{ Autres résineux } & \multirow{3}{*}{ Départements } \\
\hline \multicolumn{2}{|c|}{$\begin{array}{l}\text { Prlx mayens } \\
\qquad(\mathrm{F} / \mathrm{m} 3)\end{array}$} & \multirow{2}{*}{$\begin{array}{c}\begin{array}{c}\text { volume } \\
\text { vendu } \\
\text { (m3) }\end{array} \\
\overline{\bar{\sigma}} \\
\stackrel{5}{\circ}\end{array}$} & \multicolumn{2}{|c|}{$\begin{array}{l}\text { Prix moyens } \\
(\mathrm{F} / \mathrm{m} 3)\end{array}$} & \multirow{2}{*}{$\begin{array}{c}\begin{array}{c}\text { Volume } \\
\text { vendu } \\
\text { (m3) }\end{array} \\
\overline{\widetilde{J}} \\
\stackrel{5}{\circ}\end{array}$} & \multicolumn{2}{|c|}{$\begin{array}{l}\text { Prix moyens } \\
(\mathrm{F} / \mathrm{m} 3)\end{array}$} & \multirow{2}{*}{$\begin{array}{c}\begin{array}{c}\text { Volume } \\
\text { vendu } \\
\text { (m3) }\end{array} \\
\overline{\frac{\pi}{0}} \\
\end{array}$} & \multicolumn{2}{|c|}{$\begin{array}{l}\text { Prix moyens } \\
(\mathrm{F} / \mathrm{m} 3)\end{array}$} & \multirow{2}{*}{$\begin{array}{c}\begin{array}{c}\text { Volume } \\
\text { vendu } \\
\text { (m3) }\end{array} \\
\overline{\text { 。 }} \\
\end{array}$} & \\
\hline $\begin{array}{l}+ \\
\text { వ } \\
\text { ปิ }\end{array}$ & 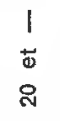 & & $\begin{array}{l}+ \\
+ \\
\text { ' }\end{array}$ & $\begin{array}{l}\text { I } \\
\pm \\
\text { ని }\end{array}$ & & $\begin{array}{l}+ \\
+ \\
\mathbb{0}\end{array}$ & $\begin{array}{l}1 \\
\pm \\
\text { 요 }\end{array}$ & & \begin{tabular}{l}
+ \\
\pm \\
\multirow{2}{*}{}
\end{tabular} & 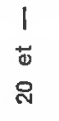 & & \\
\hline 111 & 28 & 15200 & 42 & 10 & 2100 & - & - & - & 64 & 10 & 2600 & 1 Ain \\
\hline- & - & 500 & - & - & 300 & - & - & - & - & - & 500 & 2 Aisne \\
\hline 158 & 11 & 600 & 78 & 8 & 4900 & - & - & 100 & - & - & 400 & 3 Allier \\
\hline 34 & 21 & 7100 & 35 & 16 & 17800 & - & - & - & 34 & 16 & 31500 & 4 Basses-Alpes \\
\hline 63 & 11 & 1400 & 36 & 11 & 8800 & - & - & - & 46 & 15 & 42100 & 5 Hautes-Alpes \\
\hline 81 & 14 & 5300 & 39 & 13 & 5000 & - & - & - & 65 & 10 & 900 & 6 Alpes-Maritimes \\
\hline 87 & 43 & 8400 & 34 & 18 & 2900 & - & - & 200 & 40 & 19 & 2300 & 7 Ardèche \\
\hline 70 & 15 & 3600 & 25 & 8 & 1500 & - & - & - & - & - & 300 & 8 Ardennes \\
\hline 73 & 18 & 1500 & 60 & 13 & 2300 & - & - & - & 67 & 19 & 1600 & 9 Ariège \\
\hline - & - & 300 & 69 & - & 1200 & - & - & - & 47 & 9 & 1900 & 10 Aube \\
\hline - & - & 400 & 69 & 25 & 2400 & - & - & - & 85 & 28 & 4300 & 11 Aude \\
\hline- & - & 300 & 41 & 12 & 2000 & 一 & - & - & 72 & 11 & 1000 & 12 Aveyron \\
\hline- & - & 一 & - & - & - & - & - & - & 32 & 18 & 600 & 13 B.-du-Rhône \\
\hline - & - & - & 100 & - & 900 & - & - & - & - & - & - & 14 Calvados \\
\hline 121 & 15 & 2300 & 53 & 10 & 8300 & - & - & 600 & - & - & 600 & 15 Cantal \\
\hline- & - & - & - & - & 300 & 46 & 24 & 1000 & - & - & - & 16 Charente \\
\hline - & - & - & - & - & - & 39 & 18 & 10300 & - & - & 一 & 17 Char.-Maritime \\
\hline- & - & - & 77 & 2 & 3500 & - & - & 400 & - & - & 100 & 18 Cher \\
\hline - & - & - & 49 & 18 & 1300 & - & - & - & - & - & - & 19 Corrèze \\
\hline 一 & - & - & - & - & - & 76 & 20 & 3000 & 101 & 37 & 21300 & 20 Corse \\
\hline 78 & 26 & 2100 & 37 & 22 & 2800 & - & - & - & - & - & 700 & 21 Côte-d'Or \\
\hline- & - & - & - & - & 100 & 45 & 12 & 2300 & 一 & - & - & 22 Côtes-du-Nord \\
\hline- & 一 & 500 & 46 & 20 & 2100 & - & - & 200 & - & - & - & 23 Creuse \\
\hline 一 & - & - & - & - & - & 一 & 一 & - & - & - & - & 24 Dordogne \\
\hline 121 & 41 & 53000 & 64 & 21 & 1400 & - & - & 一 & 50 & 18 & 1000 & 25 Doubs \\
\hline 92 & 27 & 9100 & 15 & 7 & 17000 & - & - & - & 17 & 8 & 24700 & 26 Drôme \\
\hline - & - & 一 & 100 & 23 & 14800 & 一 & - & - & - & - & - & 27 Eure \\
\hline - & - & - & 87 & 12 & 1100 & - & - & - & - & - & - & 28 Eure-et-Loir \\
\hline 一 & - & - & - & - & - & - & - & 一 & - & - & - & 29 Finistère \\
\hline 72 & 18 & 5600 & 46 & 8 & 6700 & 30 & 7 & 1900 & 44 & 12 & 6400 & 30 Gard \\
\hline 一 & - & 400 & 104 & 16 & 1100 & 51 & 16 & 1100 & 66 & 16 & 800 & 31 Haute-Garonne \\
\hline 一 & - & - & - & - & 一 & 一 & - & 一 & - & 一 & - & 32 Gers \\
\hline
\end{tabular}

3. Aucune indication de prix n'est donnée lorsque les volumes vendus sont trop falbles.

4. Le peuplier n'est pas comprls dans ces statistiques. II en a été vendu $16800 \mathrm{~m} 3$, principalement dans les réglons de Picardie, Aquitaine, Rhóne-Alpes, Nord et Bourgogne. 
Prix moyens et volumes des bois

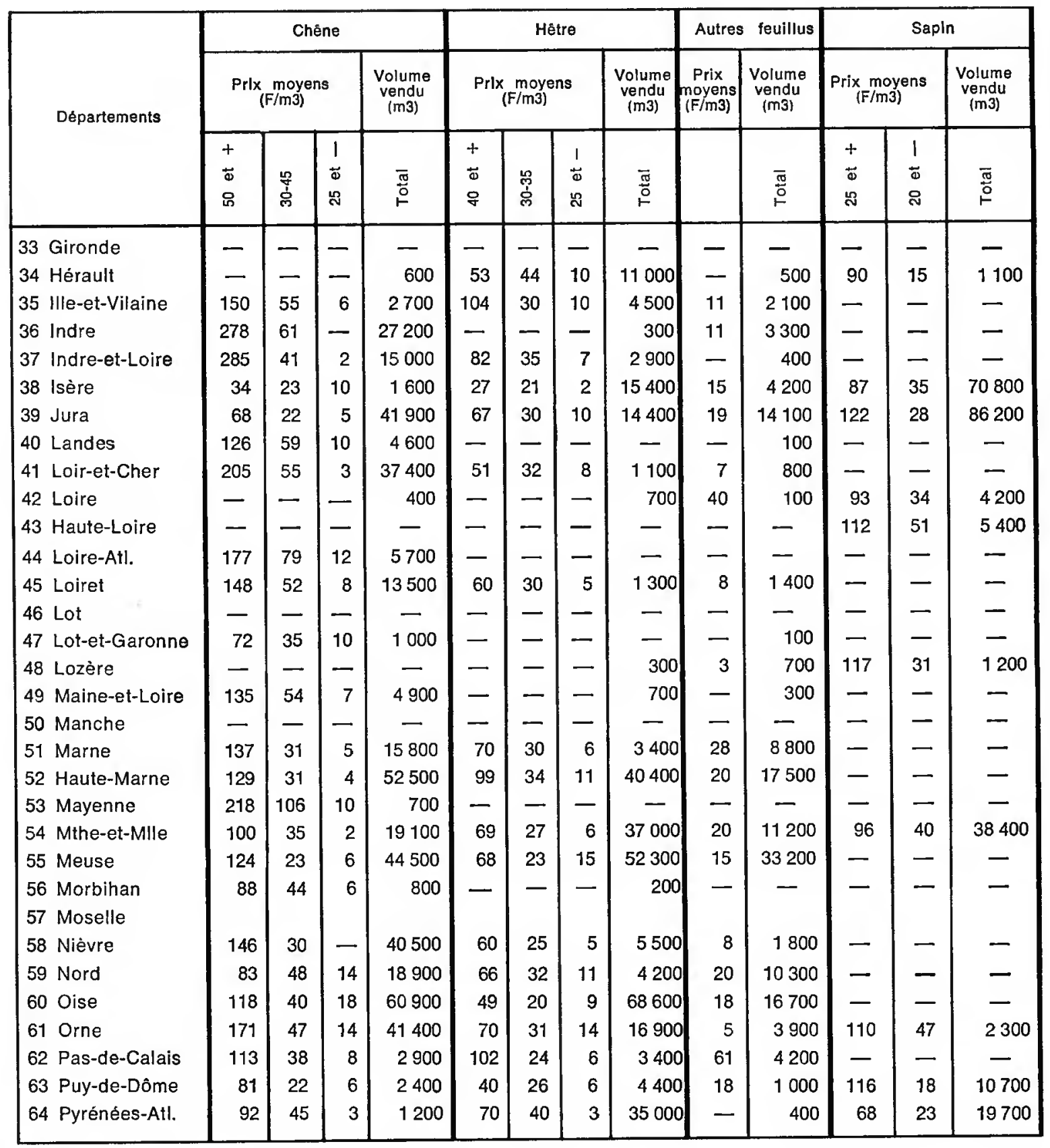

\section{Remarques}

1. Les prix moyens s'entendent taxe forfaitaire exclue.

2. Les prix moyens et volumes vendus ne sont calculés que pour les volumes-tiges Inventoriés. 
sur pied vendus aux Grandes Ventes 1970

\begin{tabular}{|c|c|c|c|c|c|c|c|c|c|c|c|c|}
\hline \multicolumn{3}{|c|}{ Eplcéa } & \multicolumn{3}{|c|}{ PIn sylvestre } & \multicolumn{3}{|c|}{ PIn maritime } & \multicolumn{3}{|c|}{ Autres résineux } & \multirow{3}{*}{ Dépsitements } \\
\hline \multicolumn{2}{|c|}{$\begin{array}{l}\text { Prlx moyens } \\
\qquad(\mathrm{F} / \mathrm{m} 3)\end{array}$} & \multirow{2}{*}{ 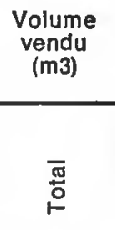 } & \multicolumn{2}{|c|}{$\begin{array}{l}\text { Prix moyens } \\
\qquad(\mathrm{F} / \mathrm{m} 3)\end{array}$} & \multirow{2}{*}{$\begin{array}{c}\begin{array}{c}\text { Volume } \\
\text { vendu } \\
(\mathrm{m} 3)\end{array} \\
\overline{\mathrm{m}} \\
\overline{\mathrm{\sigma}} \\
⺊\end{array}$} & \multicolumn{2}{|c|}{$\begin{array}{l}\text { Prix moyens } \\
(\mathrm{F} / \mathrm{m} 3)\end{array}$} & \multirow{2}{*}{$\begin{array}{c}\begin{array}{c}\text { Volume } \\
\text { vendu } \\
\text { (m3) }\end{array} \\
\overline{\bar{\Xi}} \\
\text { 范 }\end{array}$} & \multicolumn{2}{|c|}{$\begin{array}{l}\text { Prix moyens } \\
{ }_{(\mathrm{F} / \mathrm{m} 3)}\end{array}$} & \multirow{2}{*}{$\begin{array}{c}\begin{array}{c}\text { Volume } \\
\text { vendu } \\
\text { (m3) }\end{array} \\
\overline{\frac{\Phi}{0}}\end{array}$} & \\
\hline $\begin{array}{l}+ \\
\Phi \\
\Phi\end{array}$ & $\begin{array}{l}1 \\
\pm \\
\mathbb{N}\end{array}$ & & $\begin{array}{l}+ \\
\vec{\Phi} \\
\stackrel{\omega}{a}\end{array}$ & $\begin{array}{l}1 \\
\pm \\
\text { న }\end{array}$ & & 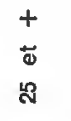 & 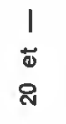 & & 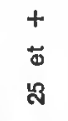 & 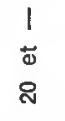 & & \\
\hline- & - & - & - & - & - & 40 & 16 & 98600 & 一 & - & - & 33 Gironde \\
\hline 90 & 15 & 2300 & - & 一 & 一 & - & - & - & 30 & 12 & 3900 & 34 Hérault \\
\hline 一 & - & 100 & 90 & 12 & 10500 & - & - & - & - & - & 100 & 35 Ille-et-Vilaine \\
\hline- & - & - & 53 & 4 & 6400 & 65 & - & 1500 & - & - & - & 36 Indre \\
\hline 一 & 一 & 一 & 77 & 18 & 11100 & 83 & 18 & 3000 & - & - & 一 & 37 Indre-et-Loire \\
\hline 88 & 33 & 64400 & 一 & - & 500 & - & - & 200 & 27 & 9 & 1700 & 38 Isère \\
\hline 121 & 27 & 24500 & 68 & 18 & 2200 & 一 & 一 & - & 41 & 14 & 1500 & 39 Jura \\
\hline 一 & - & - & - & - & - & 45 & 16 & 133600 & - & - & - & 40 Landes \\
\hline- & 一 & 一 & 85 & 20 & 12100 & 一 & 一 & 一 & - & 一 & - & 41 Loir-et-Cher \\
\hline 94 & 34 & 6500 & 59 & 27 & 11100 & - & 一 & - & - & - & 600 & 42 Loire \\
\hline 120 & 52 & 10100 & 57 & 26 & 7900 & - & - & - & - & - & 600 & 43 Haute-Loire \\
\hline - & 一 & - & 一 & 一 & - & - & 一 & 一 & - & 一 & - & 44 Loire-Atl. \\
\hline - & - & 一 & 92 & 22 & 54900 & - & 一 & - & - & - & 100 & 45 Loiret \\
\hline 一 & - & - & - & - & - & 一 & 一 & 一 & 一 & - & - & 46 Lot \\
\hline- & - & - & - & - & - & 30 & 18 & 2000 & - & - & 200 & 47 Lot-et-Garonne \\
\hline 70 & 24 & 3100 & 48 & 23 & 14400 & 40 & 25 & 1300 & 50 & 23 & 20500 & 48 Lozére \\
\hline- & - & - & 75 & 25 & 1500 & 67 & 22 & 2400 & 一 & - & - & 49 Maine-et-Loire \\
\hline- & - & - & - & - & 一 & 一 & - & - & - & - & - & 50 Manche \\
\hline 40 & 10 & 2900 & 40 & 8 & 1800 & - & - & - & 一 & - & 700 & 51 Marne \\
\hline 一 & - & 600 & 57 & 19 & 2000 & - & 一 & 一 & 53 & 19 & 1900 & 52 Haute-Marne \\
\hline- & 一 & - & - & - & - & - & - & - & 一 & - & 一 & 53 Mayenne \\
\hline 79 & 37 & 5500 & 48 & 12 & 3400 & - & 一 & - & 19 & 9 & 3600 & 54 Mthe-et-Mile \\
\hline 64 & 35 & 3200 & 31 & 15 & 3400 & 一 & - & - & 14 & 16 & 12600 & 55 Meuse \\
\hline - & 一 & - & 79 & 13 & 2200 & 一 & 一 & 一 & 一 & 一 & 一 & $\begin{array}{l}56 \text { Morbihan } \\
57 \text { Moselle }\end{array}$ \\
\hline 一 & 一 & - & 一 & 一 & 600 & - & 一 & - & - & - & 300 & 58 Nièvre \\
\hline - & 一 & 一 & 51 & 24 & 1500 & - & - & - & - & - & - & 59 Nord \\
\hline 一 & - & 一 & 69 & 11 & 11800 & - & 一 & 500 & - & - & 400 & 60 Oise \\
\hline 95 & 44 & 3200 & 109 & 36 & 34000 & - & - & 300 & - & - & - & 61 Orne \\
\hline- & - & - & 一 & 一 & - & - & 一 & 一 & 一 & 一 & - & 62 Pas-de-Calais \\
\hline 116 & 15 & 20200 & 78 & 10 & 20200 & - & 一 & - & 一 & 一 & 300 & 63 Puy-de-Dôme \\
\hline - & - & - & - & - & 300 & 37 & 11 & 800 & - & - & 200 & 64 Pyrénées-Atl. \\
\hline
\end{tabular}

3. Aucune indication de prlx n'est donnée lorsque les volumes vendus sont trop falbles.

4. Le peuplier n'est pas compris dans ces statlstlques. II en a été vendu $16800 \mathrm{~m} 3$, princlpalement dans les régions de Picardie, Aquitaine, Rhóne-Alpes, Nord ot Bourgogne. 


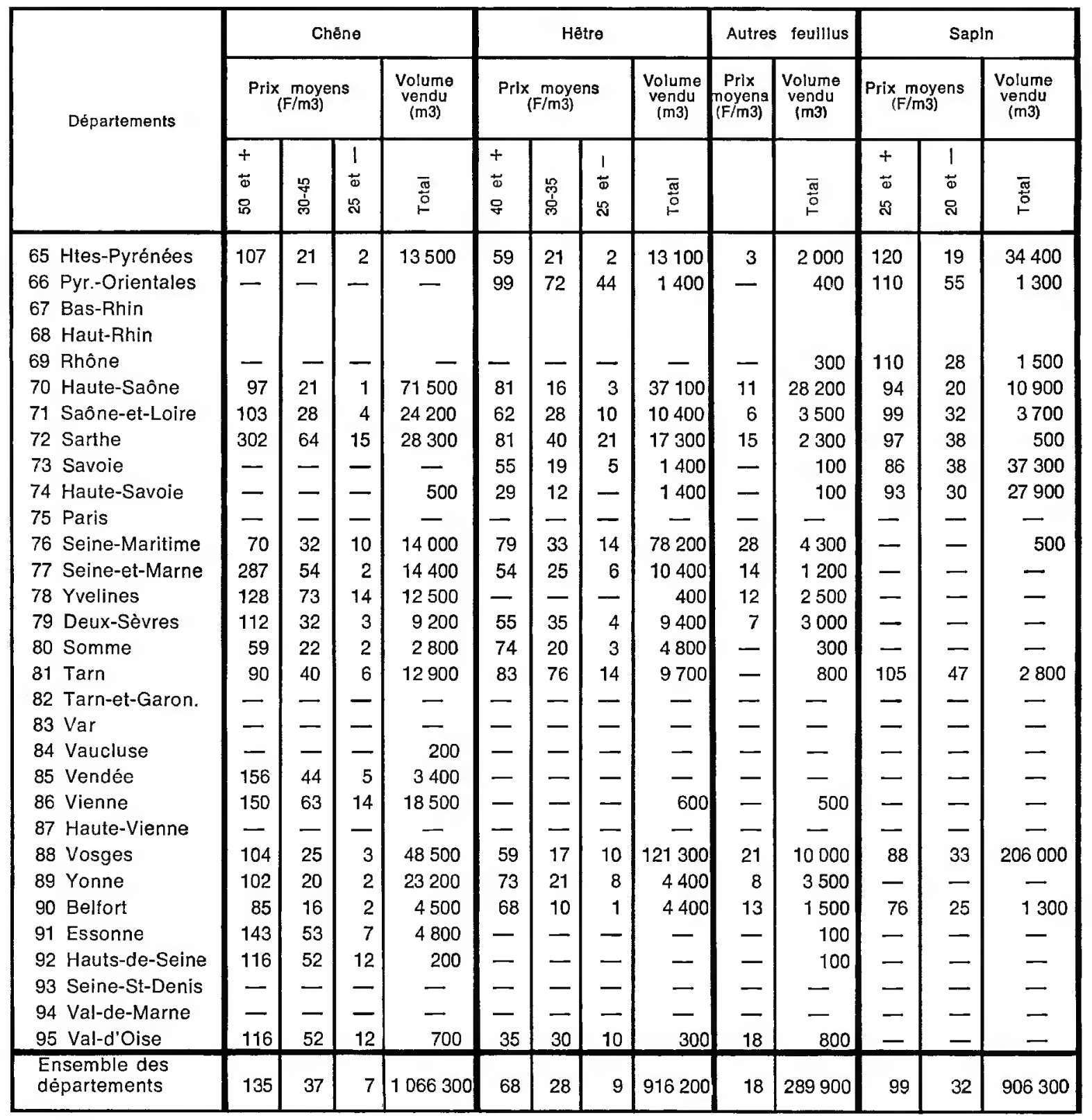

Remarques

1. Les prix moyens s'entendent taxe forfaitaire exclue.

2. Les prix moyens et volumes vendus ne sont calculés que pour les volumes-tiges Inventoriés. 
sur pied vendus aux Grandes Ventes 1970

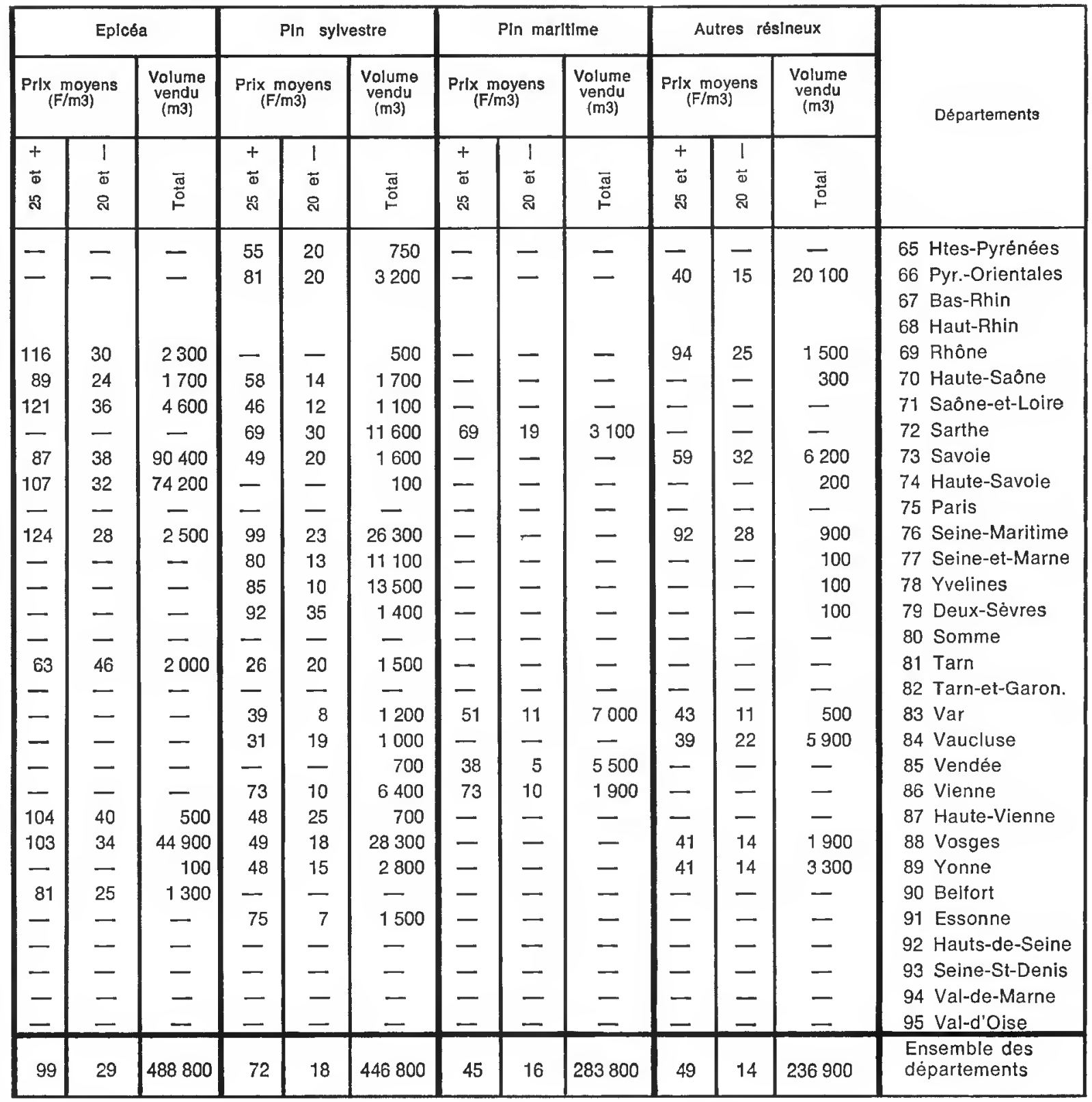

3. Aucune indication de prix n'est donnée lorsque les volumes vendus sont trop falbles.

4. Le peupljer n'est pas compris dans ces statistiques. II en a été vendu $16800 \mathrm{m3}$, princlpalement dans les réglons de Picardie, Aquitaine, Rhône-Alpes, Nord et Bourgogne. 


\section{ANNEXE IV}

\section{RÉSULTATS COMPARÉS DES GRANDES VENTES DEPUIS 10 ANS}

\begin{tabular}{|c|c|c|c|c|c|c|}
\hline Années & Catégories (1) & $\begin{array}{l}\text { Volume } \\
\text { mis en } \\
\text { vente } \\
\left(1000 \mathrm{~m}^{3}\right)\end{array}$ & $\begin{array}{l}\text { Volume } \\
\text { vendu } \\
\left(1000 \mathrm{~m}^{3}\right)\end{array}$ & $\begin{array}{l}\text { Volume } \\
\text { invendu } \\
\left(1000 \mathrm{~m}^{3}\right)\end{array}$ & $\begin{array}{c}\% \\
\text { invendu }\end{array}$ & $\begin{array}{c}\text { Recettes } \\
\text { (millions } \\
\text { de F } \\
\text { courants) } \\
\text { (2) }\end{array}$ \\
\hline 1960 & $\begin{array}{l}\text { Feuillus } \ldots \ldots \\
\text { Résineux } \ldots \ldots \\
\text { Total } \ldots \ldots \ldots\end{array}$ & $\begin{array}{l}4017 \\
1962 \\
5979\end{array}$ & $\begin{array}{l}3096 \\
1915 \\
5008\end{array}$ & $\begin{array}{r}924 \\
47 \\
971\end{array}$ & $\begin{aligned} & 23 \% \\
& 2 \% \\
& 16 \%\end{aligned}$ & 195,2 \\
\hline 1961 & $\begin{array}{l}\text { Feuillus } \ldots \ldots \ldots \\
\text { Résineux } \ldots \ldots \ldots \\
\text { Total } \ldots \ldots \ldots \ldots\end{array}$ & $\begin{array}{l}4054 \\
2307 \\
6361\end{array}$ & $\begin{array}{l}3575 \\
2210 \\
5785\end{array}$ & $\begin{array}{r}479 \\
97 \\
576\end{array}$ & $\begin{array}{r}12 \% \\
4 \% \\
9 \%\end{array}$ & 241,1 \\
\hline 1962 & $\begin{array}{l}\text { Feuillus } \ldots \ldots \ldots \\
\text { Résineux } \ldots \ldots \ldots \\
\text { Total } \ldots \ldots \ldots \ldots\end{array}$ & $\begin{array}{l}4188 \\
2401 \\
6589\end{array}$ & $\begin{array}{l}3363 \\
2098 \\
5461\end{array}$ & $\begin{array}{r}825 \\
303 \\
1128\end{array}$ & $\begin{array}{l}20 \% \\
13 \% \\
17 \%\end{array}$ & 216,1 \\
\hline 1963 & $\begin{array}{l}\text { Feuillus } \ldots \ldots \ldots \\
\text { Résineux } \quad \ldots \ldots \\
\text { Total } \ldots . . .\end{array}$ & $\begin{array}{l}4014 \\
2477 \\
6491\end{array}$ & $\begin{array}{l}3357 \\
2124 \\
5481\end{array}$ & $\begin{array}{r}657 \\
353 \\
1010\end{array}$ & $\begin{array}{l}16 \% \\
14 \% \\
16 \%\end{array}$ & 225,8 \\
\hline 1964 & $\begin{array}{l}\text { Feuillus } \ldots \ldots \ldots \\
\text { Résineux } \ldots \ldots \\
\text { Total } \ldots \ldots \ldots\end{array}$ & $\begin{array}{l}3970 \\
2473 \\
6443\end{array}$ & $\begin{array}{l}3330 \\
2137 \\
5467\end{array}$ & $\begin{array}{l}640 \\
336 \\
976\end{array}$ & $\begin{array}{l}16 \% \\
14 \% \\
15 \%\end{array}$ & 227,8 \\
\hline 1965 & $\begin{array}{l}\text { Feuillus } \ldots \ldots \ldots \\
\text { Résineux } \quad \ldots \ldots \\
\text { Total } \ldots \ldots \ldots\end{array}$ & $\begin{array}{l}4181 \\
2767 \\
6948\end{array}$ & $\begin{array}{l}3433 \\
2340 \\
5773\end{array}$ & $\begin{array}{r}748 \\
427 \\
1175\end{array}$ & $\begin{array}{l}18 \% \\
15 \% \\
17 \%\end{array}$ & 247,4 \\
\hline 1966 & $\begin{array}{l}\text { Feuillus } \\
\text { Résineux } \ldots \ldots \ldots \\
\text { Total } \ldots \ldots \ldots\end{array}$ & $\begin{array}{l}4104 \\
2847 \\
6951\end{array}$ & $\begin{array}{l}3177 \\
2131 \\
5308\end{array}$ & $\begin{array}{r}927 \\
716 \\
1643\end{array}$ & $\begin{array}{l}23 \% \\
25 \% \\
24 \%\end{array}$ & 217,3 \\
\hline 1967 & $\begin{array}{l}\text { Feuillus } \ldots \ldots \ldots \\
\text { Résineux } \quad \ldots \ldots \\
\text { Total } \ldots \ldots \ldots\end{array}$ & $\begin{array}{l}3826 \\
2335 \\
6161\end{array}$ & $\begin{array}{l}2691 \\
1631 \\
4322\end{array}$ & $\begin{array}{r}1135 \\
704 \\
1839\end{array}$ & $\begin{array}{l}29 \% \\
30 \% \\
29 \%\end{array}$ & 158,8 \\
\hline 1968 & $\begin{array}{l}\text { Feuillus } \ldots \ldots \ldots \\
\text { Résineux } \ldots \ldots \\
\text { Total } \ldots \ldots \ldots\end{array}$ & $\begin{array}{l}4109 \\
2614 \\
6723\end{array}$ & $\begin{array}{l}3201 \\
2130 \\
5331\end{array}$ & $\begin{array}{r}908 \\
484 \\
1392\end{array}$ & $\begin{array}{l}22 \% \\
18 \% \\
21 \%\end{array}$ & 202,5 \\
\hline 1969 & $\begin{array}{l}\text { Feuillus } \ldots \ldots \ldots \\
\text { Résineux } \ldots \ldots \ldots \\
\text { Total } \ldots \ldots \ldots \ldots\end{array}$ & $\begin{array}{l}4133 \\
2952 \\
7085\end{array}$ & $\begin{array}{l}3696 \\
2657 \\
6353\end{array}$ & $\begin{array}{l}437 \\
295 \\
732\end{array}$ & $\begin{array}{l}11 \% \\
10 \% \\
10 \%\end{array}$ & 304,3 \\
\hline 1970 & $\begin{array}{l}\text { Feuillus } \ldots \ldots \ldots \\
\text { Résineux } \ldots \ldots \ldots \\
\text { Total } \ldots \ldots \ldots \ldots\end{array}$ & $\begin{array}{l}4321 \\
2884 \\
7205\end{array}$ & $\begin{array}{l}3718 \\
2553 \\
6271\end{array}$ & $\begin{array}{l}603 \\
331 \\
934\end{array}$ & $\begin{array}{l}14 \% \\
11 \% \\
13 \%\end{array}$ & 342,1 \\
\hline
\end{tabular}

(1) Les volumes de feuillus et résineux s'entendent toutes catégories comprises.

(2) Les recettes s'entendent charges et taxe forfaitaire incluses. 